\title{
TERRESTRIAL LASER SCANNER FOR MONITORING THE DEFORMATIONS AND THE DAMAGES OF BUILDINGS
}

\author{
G. Vacca ${ }^{a}$, F. Mistretta ${ }^{a}$, F. Stochino ${ }^{a}$, A. Dessi ${ }^{a}$ \\ ${ }^{a}$ DICAAR, Dep. of Civil and Environmental Engineering and Architecture, University of Cagliari, P.zza D'Armi 09123 Cagliari - \\ (vaccag, fmistret)@unica.it
}

Commission V, WG V/3

KEY WORDS: 3D modelling, deformations measurements, terrestrial laser scanner

\begin{abstract}
:
The paper presents the use of the terrestrial laser scanner for the study and the assessment of damaged buildings. The terrestrial laser scanner provides the ability to detect the geometric 3D model of a building without any physical contact with the structure. Knowledge of the 3D model will give the opportunity to study the deformation and quantify the damages.

Three case studies are presented relating to damaged and/or unsafe buildings: Sivillier Castle (Villasor - Sardinia- Italy), the Bell Tower of Mores (Sardinia-Italy) and industrial building (Cagliari - Italy).

The first two cases concern buildings of historical and architectural importance that present a state of compromised conservation; the last, an industrial building compromised by fire. In all cases, a laser scanner survey was carried out that not only provided valuable information but also highlighted structural metric deformation and degradation.
\end{abstract}

\section{INTRODUCTION}

The monitoring of building structures have an increasingly important role in the engineering field, above all because they are concerned with the impact that such structures have in the area where they were built. Often, when walking through the old town centers, we realize just how obselete and dangerous some buildings (even historic-cultural ones) are. The interest of some local governments in this problem has led, in the last few years, to the study and the trying out of measuring and monitoring methods which, quickly and at low cost, allow to define the extent of the deformation and the degrade in an accurate and reliable way. The most frequent cases of monitoring and control can be classified as follows: verification of the deformation and damage caused by natural calamities (e.g. earthquakes), or malicious (e.g. fires); verification of the degrade caused by weather conditions; verification of the present precarious state of a structure with respect to its initial project; verification of the result of bad workmanship. The requirement, therefore, is to identify techniques that are able to carry out accurate and reliable measuring of structural deformation, and that are easy to obtain and are not too expensive. Moreover, in case of the unstable buildings, especially if this are historic and cultural buildings, instruments are required that do not make direct contact with the structure itself. Among all the geomatic techniques, that have some of these characteristics, there are the following: measurement with Total Stations, measurement with GNSS technology, close range photogrammetry and Terrestrial Laser Scanning. There follows a brief description of these techniques:

1. Classical survey with Total Stations is widespread for the accurate measuring of a limited number of accessible or inaccessible points. The drawback of this technique, when used for monitoring structures, is the long time, above all for complex structures in which a high number of points must be surveyed in order to obtain accurate information regarding the structure and its deformation.
2.The Global Navigation Satellite System (GNSS) is a technique that is now usually used to monitor the condition of structures and buildings [2-6], also in combination with other sensors like accelerometers, or inertial navigation systems [11]; but this technique can't to use to carry out measurements indoor or in presence of obstruct.

3. Digital close-range photogrammetry has been a low cost alternative [15], and is highly accurate [9]. It also offers a quick, remote, three-dimensional (3D) data acquisition with images that provide a permanent visual recording of the test. But the compulsory use of targets might be disadvantageous in some circumstances; especially when the access to the object is risky or even when it is inaccessible to operators. In contrast, the depth dimension could be slightly solved, just avoiding the use of multiple convergent images.

4. Terrestrial laser scanning (TLS) has become a new alternative to health monitoring of structures incorporating novelty approaches [11] and computer methods [13].

The approach proposed in this paper is one of studying the potential of Terrestrial Laser Scanning (TLS) in terms of monitoring structures and buildings that have been damaged by natural calamities or by malicious intent. Three cases were presented. Firstly, the study of the deformation of some vertical walls of Sivillier Castle (XV Century). Secondly, the study of the verticality of a Bell Tower (XIX Century), $40 \mathrm{~m}$ high, and the degrade of the statue at the top of it. Thirdly, the study of the deformation of a prefabricated structure relative to an industrial building which had been the object of arson.

\section{TERRESTRIAL LASER SCANNER (TLS)}

The scanning of an object, or a building, consists of a series of scansions of the whole building, both internally and externally. The result obtained is a multitude of points which allow for a $3 \mathrm{D}$ reconstruction of the object with high accuracy. 


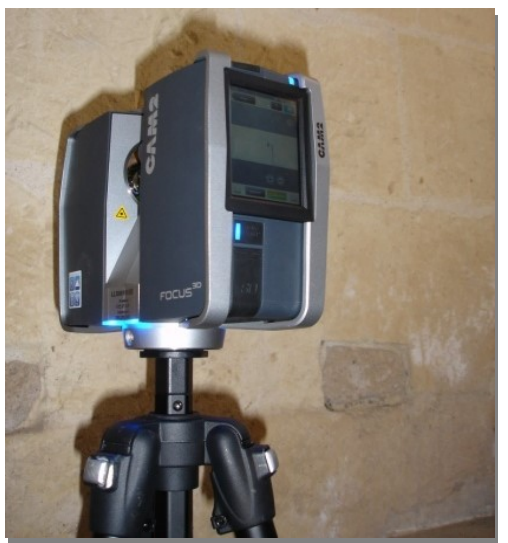

Figure 1: Focus 3D Laser scanner

The Faro 3D laser Focus (fig. 1) was used in all the cases studied presented successively. It is a compact scanner characterized by an operative range that varies between $0.6 \mathrm{~m}$ and $120 \mathrm{~m}$ with a linear distance error of $\pm 2 \mathrm{~mm}$ for scannerobject distances comprised between $10 \mathrm{~m}$ and $25 \mathrm{~m}$, and a noise (that is to say, the standard deviation of the values with respect to the best-fit plan) which varies from between $0.6 \mathrm{~mm}$ and 10 $\mathrm{mm}$ with a reflectivity of $90 \%$ and $2.2 \mathrm{~mm}$ to $25 \mathrm{~mm}$ with a reflectivity of $10 \%$. It has a vertical visual field of $305^{\circ}$ and a horizontal one of $360^{\circ}$. The vertical and horizontal resolution is $0.009^{\circ}$. It has a scanning speed of 976.000 points $/ \mathrm{sec}$, and a reduced weight. Incorporated into the laser is a color digital camera with a resolution of 70 megapixels

The laser scanning provides a point cloud with a high density points, each one of them having the coordinates $\mathrm{x}, \mathrm{y}, \mathrm{z}$, relative to an intrinsic reference system to the instrument and the reflectivity, which is indicative of the physical characteristics of the surface scanned. By way of the digital images obtained, the scansion is completed with RGB information of the object scanned; allowing for the integration of the analyses of the structure, e.g., those referring to the degrade of the building materials.

\subsection{Data processing}

The handling and processing of the point cloud was done by way of the JRC Reconstructor Software v. 3.1.0 (335), of Gexcel Ltd. Reconstructor is a software that enables the carrying out of all the processing operations of the point cloud until the $3 \mathrm{D}$ model of the structure or building scanned is obtained.

The processing to which the point cloud was subjected can be synthesized in three phases: pre-processing, pre-alignment and alignment. Once the scansions of the software have been loaded, they undergo 'cleaning' by way of the application of a series of algorithms that extract the information needed for the successive phase of data processing. With this procedure, known as pre-processing, operations are carried out which focus on noise reduction, the calculation of the normal vectors, the determining of the outlines, and the calculation of confidence; values that enable the assessment of the measurement reliability on each single point. The scanning accuracy does not only depend on the laser scanner used, but is also conditioned by such factors as the angle of incidence between the laser belt and the tangent plane at the point, the distance from the point, and the material of the object on which the laser beam strikes. The confidence value is therefore an average weighing of the values of the normal vectors on the surfaces, of the distance and the reflectivity. During the pre-processing phase, the points that go over the minimum confidence value are discarded. The point clouds treated in this way are oriented in a relative manner (one is taken as a reference, while the others are considered mobile); at first, approximately, by way of the matching of natural features, or targets in two adjacent scannings; then in an accurate manner. In order to carry out this final phase, known as aligning, the iterative ICP (Iterative Closest Point) algorithm, utilized by Reconstructor, searches for control points; that is to say, it identifies in the point cloud mobile the points that are closest to the same points in the reference point cloud. The cloud point mobile is therefore shifted iteratively towards the reference model in order to reduce the distance between the control points. When the processing phase has been concluded, the series of scannings, correctly 'squared up', is geo-referred in a single system of external reference. This can be carried out by utilizing natural coordinate targets or points in the external reference system which can be recognized in the point cloud. The calculation of the mesh constitutes the final phase that enables the transformation of the $3 \mathrm{D}$ points on a continuous surface. The derived 3D model, apart from constituting a virtual prototype of the site scanned, enables the realization of diverse products. In fact, it is possible to extract vertical and horizontal sections for graphic representation, as well as realize support orthophotos for the restitution of the graphic outputs.

\section{CASES STUDIED}

\subsection{Sivillier Castle}

Siviller Castle (fig.2) is one of the most important and representative cultural heritage in Sardinia, with late Gothic architecture. The castle was built in 1415 by Giovanni Siviller in the town of Villasor, $30 \mathrm{~km}$ from Cagliari (Italy). In 1991, it was acquired as a cultural heritage by the municipality of Villasor and, after an important restoration, it became a library and cultural centre for exhibitions and conventions. In the last few years, the castle has been in a very poor state, and the wooden structures and walls have needed restoration work to halt the progressive decay. As a part of the study for the restoration project, the building was scanned with a terrestrial laser scanner. The scanning provided detailed dimensional information of the building, and has enabled the study of the structural deformations and damage; in particular, the deformations of the bearing walls.

The scanning required an accuracy equal to a restitution scale of 1:50. To obtain this, 61 scannings were carried out with a resolution of $7 \mathrm{~mm}$. These scannings, superimposed by at least $30 \%$, enabled the realization of a $3 \mathrm{D}$ reconstruction of the castle, avoid of holes and with the accuracy required [18]. Of these 61 scannings, 3 were carried out using an airborne platform (fig. 3), with the aim of scanning the whole roof, while the remaining scannings were carried out from the ground. The airborne platform used consisted of a hydraulically controlled basket mounted on a truck. The scanner was positioned on the basket, and two operators lifted $15 \mathrm{~m}$ from the ground. To avoid harmful vibrations of the basket, that could have compromised the scannings, the following precautions were taken: truck engine and lifting motor turned off, operators quite still, and lack of wind. The scannings were processed by way of the software Reconstructor, as described in paragraph 2.2 . 


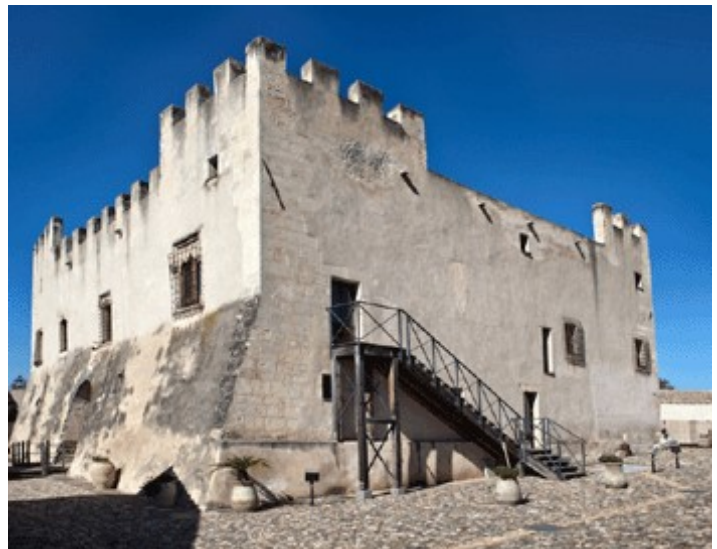

Figure 2: Sivillier Castle

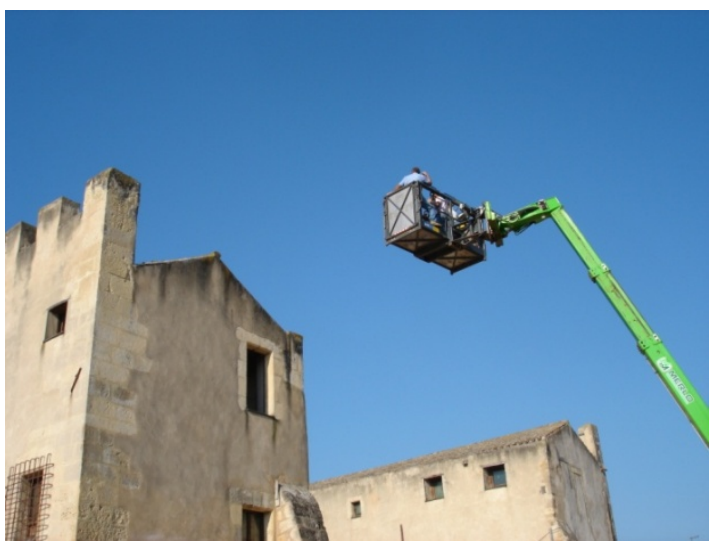

Figure 3: Airbone platform survey

The 3D model, obtained in this manner, was geo-referred in the ETRF2000, by way of utilizing natural points located in the external perimeter of the castle. The points were surveyed by way of GNSS in modality RTK; using the differential corrections from the permanent SARNET stations (http://www.geodesia.biz/sarnet/) in Sardinia. After laser scanning, and the following realization of the 3D model, it was possible to study the geometrical and structural characteristics of the castle. In particular, the deformation of the castle walls with respect to the vertical plane [7]. In fact, as can be seen in fig. 4, the vertical walls are completely out of plumb. The study of the verticality and deformations of the walls was carried out internally with Reconstructor software. First, a plane containing the $Z$ axis was generated (vertical), successively, identified the two extreme points of the facade (determined from the intersection between the plane of the facade and the horizontal one), and positioned according to the latter, the vertical plane was shifted and rotated around the $\mathrm{Z}$ axis until it contained these two points. Having defined the vertical plane of the facade in this way, the distances between the points of this plane and those of the real wall were calculated, and finally the contour line of such distances were generated, with equidistance equal to $1 \mathrm{~cm}$. With ArcGIS (ESRI) software, and starting from the contour line, the grid of the Digital Model of the Surface (DSM) was generated, with $2 \mathrm{~cm}$ pixel, and successively classified in order to obtain the verticality map of the wall. The paper reports only the study of two walls - North-West and North-East. The image of the North-West facade is shown in fig. 4, 5 and 6 instead, respectively show the contour line of the distances of the real wall - vertical plane and DSM of the verticality for the internal wall of the courtyard. In fig. 7, 8 and 9 are shown the same study for the North-East wall. In both cases, as can be inferred from the verticality DSM, the walls have a geometrical pattern which is completely different and anomalous with respect to the vertical plane, and little inclined towards any structural geometrical plan. The North-West wall has a part, at the top on the right, which is completely out of plumb, with a difference of as much as $40 \mathrm{~cm}$ from the vertical plane. The same can be said of the rest and greater part of the wall with, even in this case, receding displacements of $40 \mathrm{~cm}$ from the vertical plane. Regarding the North-East wall is concerned, the situation is similar, even if the pattern is completely different. The higher part has receding displacements from the vertical plane of up to $35 \mathrm{~cm}$, while the lower part is out of plumb by up to $15 \mathrm{~cm}$. In fig. 5 and 8 , the green contour line represent the receding part; the red, the out of plumb; the black, the intersection of the vertical plane with the wall in question.

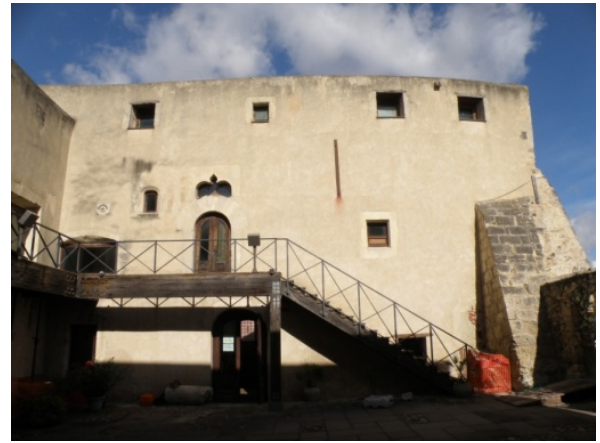

Figure 4: North-West Facade

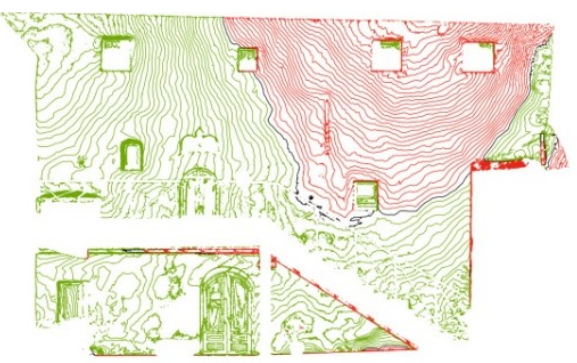

Figure 5: Contour line (North-West)

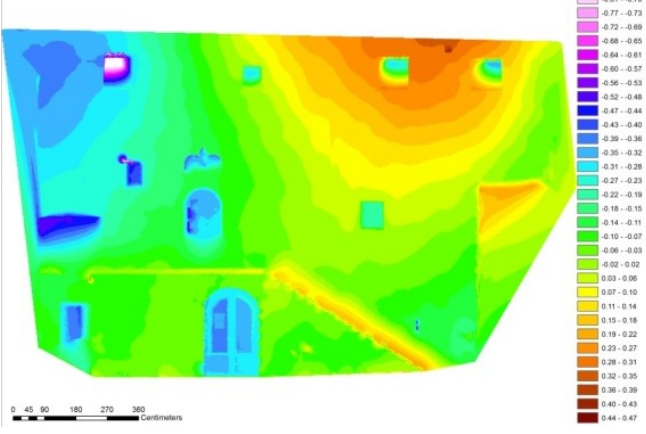

Figure 6: Verticality DSM (North-West) 


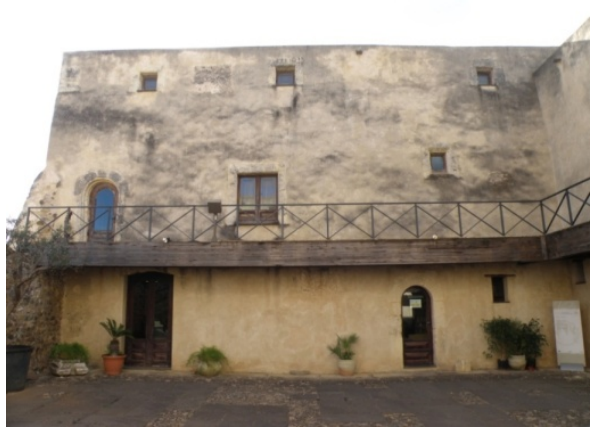

Figure 7: North-East Facade

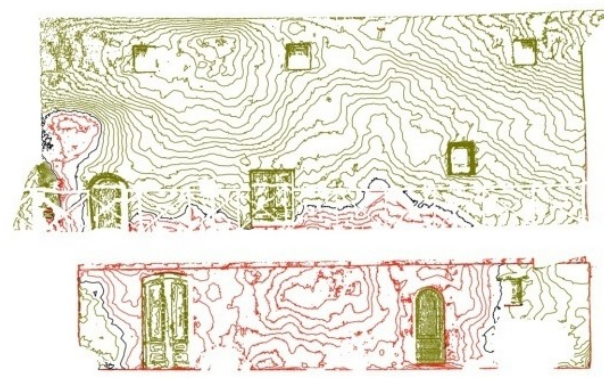

Figure 8: Contour line (North-East)

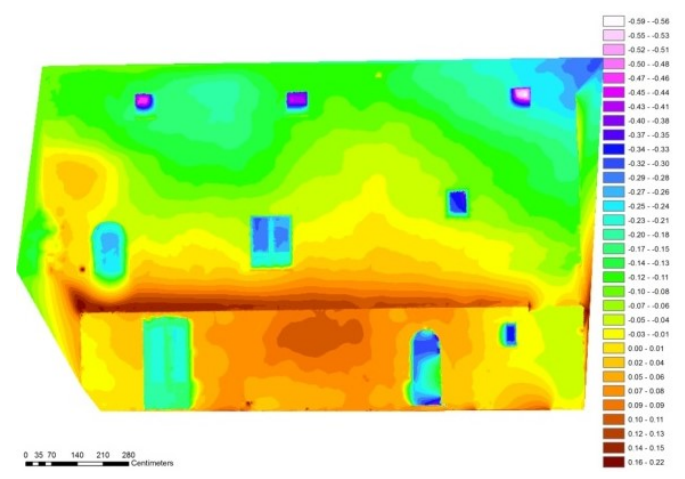

Figure 9: Verticality DSM (North-East)

\subsection{The Bell Tower in Mores}

The church bell tower of Santa Caterina, built in 1871 by the architect Salvatore Calvia Unali, is the highest bell tower in Sardinia (about $40 \mathrm{~m}$ ), and is considered the finest example of neo-classic architecture in Sardinia. Of particular interest are the five terracotta statues of the four Evangelists and Christ the Redeemer. Over the last few years, these statues have shown a high level of deterioration. This situation convinced the Municipality of Mores that it was necessary to undertake a study for a project (involving structural engineers, geomaticians and geo-technicians) of proper restoration of the statues, as well as parts of the bell tower itself. In particular, an accurate scanning was requested. Moreover, the determining of the exact height of the bell tower and any out of plumb of it was requested. In order to carry out a complete scanning of the structure, given the considerable height of it, a Liebherr motorized crane, complete with an extendible (up to $60 \mathrm{~m}$ ) telescopic arm, was used. A laser scanner (fig. 10) was mounted on the crane arm. By operating the laser with a computerized remote control, all the parts of the bell tower were scanned at a distance of no more than $10 \mathrm{~m}$, which allowed for laser ray inclinations which were not too high. The scannings were carried out in the absence of wind and with the crane engine turned off to avoid any vibrations that would have disturbed the scanning. A total of 93 scannings were carried out, both internally and externally, with a resolution of $7 \mathrm{~mm}$. The scanning of the statues was carried out with a resolution of 3 $\mathrm{mm}$, and the laser positioned at a distance of 5 metres. Moreover, the scanning operation had been planned in such a way as to guarantee the superimposition among the scannings of at least $30 \%$.

With the Reconstructor software, the point clouds was initially pre-processed and successively, utilizing exclusively natural features (at least 5 for each pair of scannings), the point clouds were pre-aligned, and then aligned with an error margin inferior to one centimetre.

The geo-referring of the point clouds was carried out in the ETR2000 system, by way of signals which were distributed at ground level, and which were surveyed during the scannings. It was not possible to insert targets along the height of the bell tower because there were no buildings around it of a similar height. The coordinates of the points at ground level were determined by way of a GPS scanning, in RTK modality. Also in this case, the SARNET network of permanent stations was used; the network transmitting the differential corrections via Internet in the ETRF2000 reference system.

For the graphical reconstruction of the statue of Christ the Redeemer a contour level restitution, with an equidistance equal to $10 \mathrm{~cm}$, was carried out (fig. 11). A classical restitution was also carried out, in which all the cracks and deterioration present in the statues were made evident.

The creation of the 3D model enabled the carrying out of analyses aimed at determining the heights of the bell tower and the statue of Christ the Redeemer, establishing the global geometrical arrangement of the structure and, in particular, assessing the out of plumb respect of its barycentric axis. As far as the bell tower is concerned, its height was measured between the external floor area and the base of the statue, and the result was $36.32 \mathrm{~m}$. The height of the statue, measured between its base and the highest point of its halo, was $3.46 \mathrm{~m}$.

The criterion adopted to assess the out of plumb of the barycentric axis of the bell tower was that of measuring the barycentric displacement of the sections from the vertical axis and passing through to the barycenters of the base [9]. From the 3D model, was extracted one horizontal section for each meter of height, starting from a measurement of $5 \mathrm{~m}$ (base section), thus excluding the part of the bell tower that is connected to church of Santa Caterina. The adjective "Ideal" was attributed to all the intersection points of the vertical with the different sections. The pattern of the barycenters of the sections did not diverge very much from that of the vertical, with values of the distance between the ideal point and the real barycenter oscillating between $0.086 \mathrm{~m}$ and $0.002 \mathrm{~m}$. Figure 12 shows the pattern overall barycenter curve (in red) with respect to the vertical (in yellow) and figure 13 shows the planimetrical barycentric dispersion of the various sections with respect to the ideal position. 


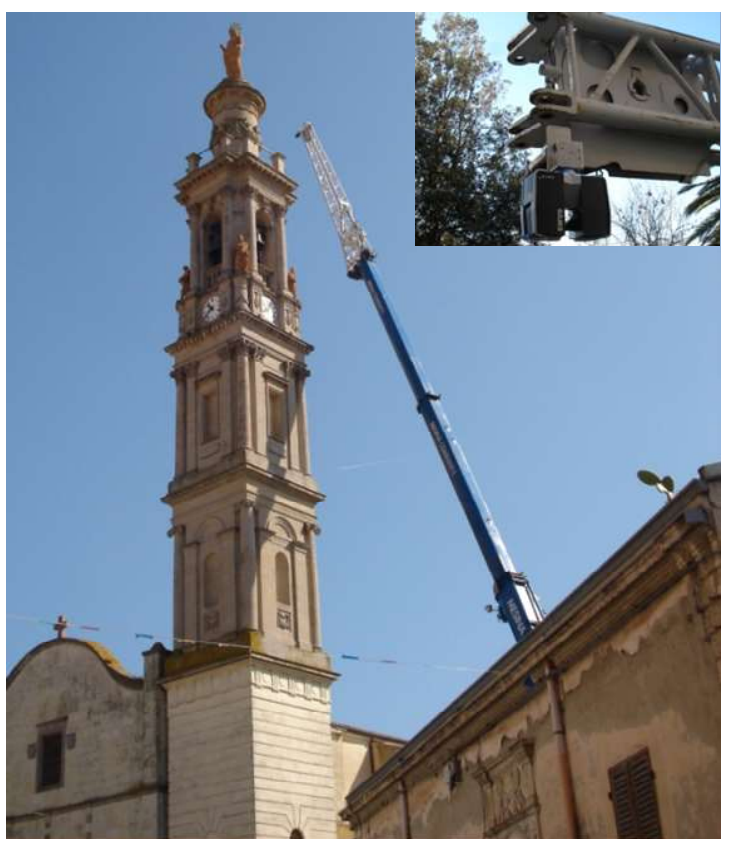

Figure 10: The Bell Tower

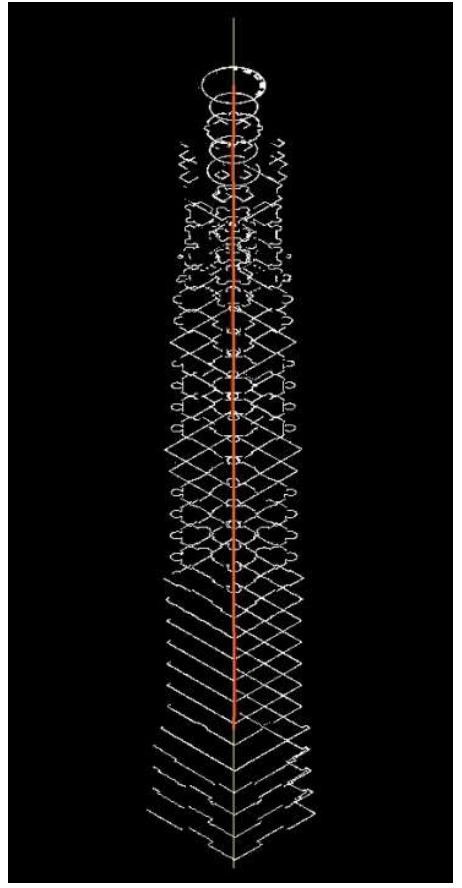

Figure 12: Position of the barycentre curve (red) respect to the vertical axis (yellow)

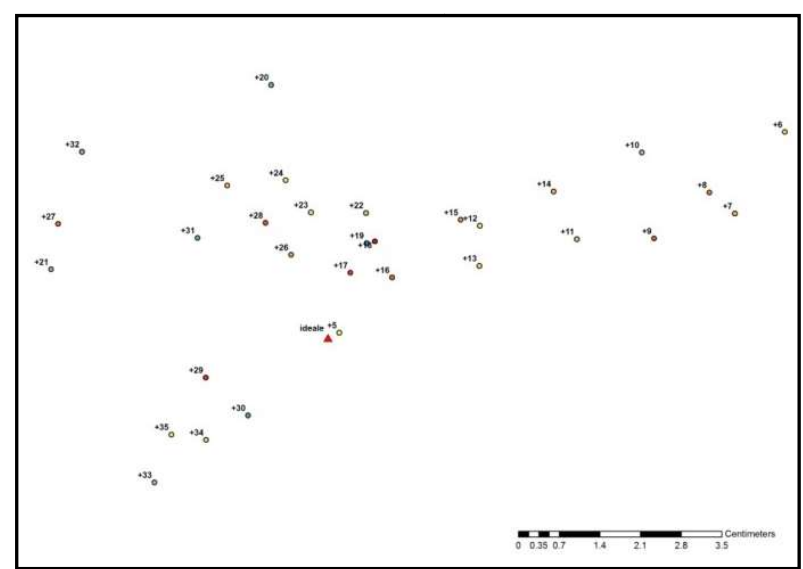

Figura 13: Planimetrical barycentric dispersion

\subsection{Industrial building}

Figure 11: Redeemer statue graphic output

The last case is that of an industrial building (Fig. 14) whose structure was partly damaged by a fire. The effects of extreme actions like blast load or fire is the subject of a current research trend (see for example [4] [5] [6] [8]), and the information obtained from a geomatic survey can be of paramount relevance in the structural analysis [2] [3]. 


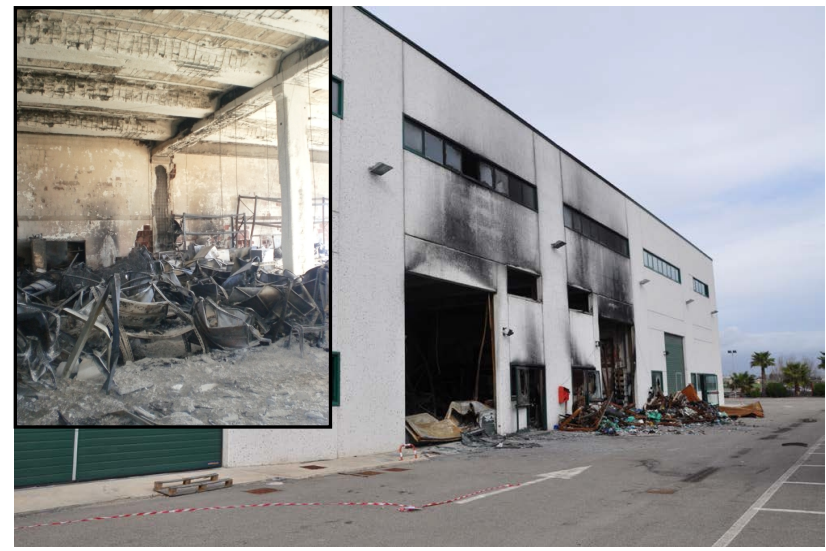

Figure 14: Industrial building

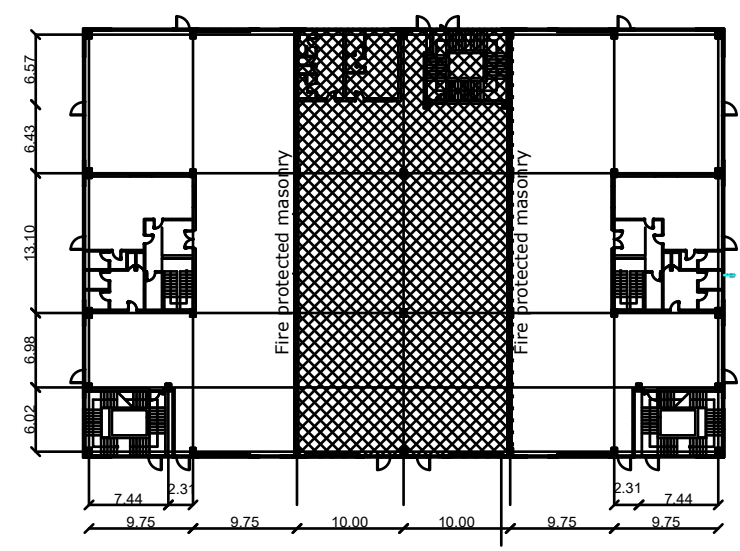

Figure 15: Zone interested by fire at the ground floor

The structural assessments of this building have been presented in [1]. The building is prefabricated and characterized by a rectangular structured plan, with a width of $40,00 \mathrm{~m}$ and a length of $60,00 \mathrm{~m}$. The building has two floors above ground: the ground floor, approximately $6,00 \mathrm{~m}$ high from the floor slab, is subdivided into three parts with the same surface area; of which, only one was directly damaged by the fire. The first floor, approximately $3,50 \mathrm{~m}$ high from the floor slab, which is not subdivided.

The building has a reinforced concrete load-bearing structure consisting of columns with rectangular cross section, precasted and prestressed beams with $\mathrm{T}$ shaped cross section which support the "Omega" shaped girders. Another kind of prestressed beams can be found in the roof structure, they are characterized by a V-shaped cross section and labelled as "Aliant beam".

The ceilings are made of prefabricated panels connected to the beams by way of completion casting.

A fire spread over the central part of the ground floor, as shown in Fig. 15, the lateral fire resistant partition walls prevented the fire from spreading to other areas of the building. Indeed, the main damage concerns the central area of the ground floor and the corresponding ceiling. For this reason the laser scanner survey was focused on these parts.

In order to assess the overall effect of the fire on the structure, (residue deformations, inclination of the columns etc.) and to determine if it has caused any geometry modifications, a TLS survey was carried out. From the 3D model obtained by the scanning, every geometrical detail of the structure was controlled; something that would have been impossible using traditional measuring methods, especially in this case in which the scanning difficulties are worsened by the deteriorated state of the structure.

In order to work on the laser scanning project, one had to consider the precision requested for the study of the deformations of the load-bearing structure of the building that had to be sub-centimetres in exactness. To this end, 19 scannings were carried out with a resolution of $7 \mathrm{~mm}$. The scanning was carried out by positioning the instrument beneath the centre lines of the longitudinal and transversal beams of the building. After carrying out the scanning and, at the same time, collecting all the RGB colour images, the data was treated through the pre-processing of each sets of points and the recording of the scanning. After finalizing the recording, errors in the order of 2-3 mm were shown. A study of the 3D model showed the deformations and damage of the columns, beams and ceiling. The graphic outputs, to be extracted from point clouds, came from the vertical sections of the longitudinal and transversal sections of the beams and the sections of the columns. With the Reconstructor software, from the single scannings (not from the 3D model) with a high level of accuracy $( \pm 2 \mathrm{~mm})$, were extracted the vertical sections of all the beams scanned, the horizontal sections of the columns, and the orthophotos relative to the structural elements being studied. As far as the study of the columns (with a total height of $6 \mathrm{~m}$ and rectangular cross section) damaged by the fire are concerned, the focus was on the variation of the centre of gravity position after the fire.

For the sake of clarity, the structural elements subjected to fire were labeled as shown in figure 16. In order to find the position of the centre of gravity, after the fire, of the six central columns (labelled 4 to 9 , see Figure 15), their geometry was recorded by the laser scanner after the fire. Obviously damage is also present on these four sections at different heights (see fig. 17 and fig. 18). The bottom and top ends of each column were not exposed to fire, and so their deformation is negligible.

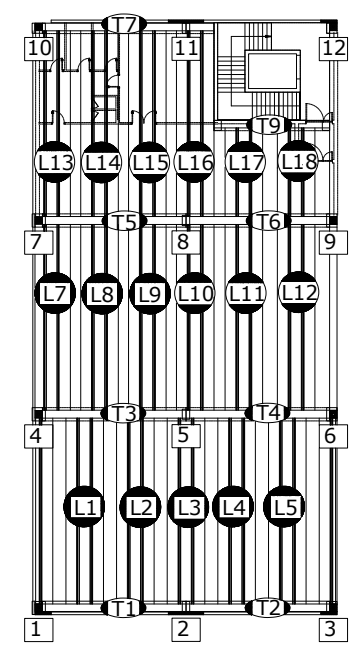

Figure 16: Structural elements scheme 


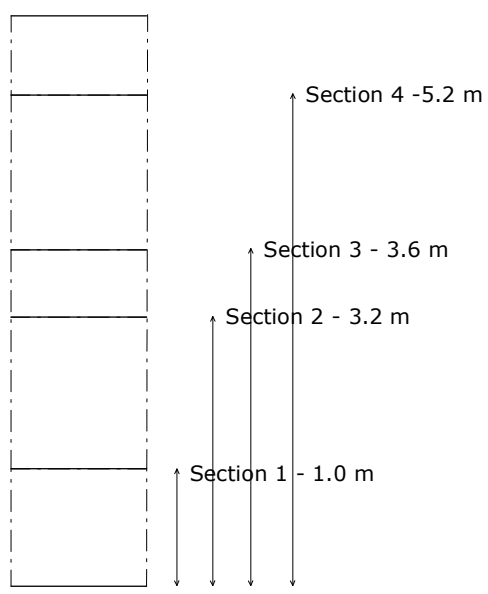

Figure 17: Scheme of the column cross-section center of gravity detection

The variation of the centre of gravity position through the column height is reported in fig. 18. Looking at this picture, it is clear that the variations of the centre of gravity position are localized in the central part of the column (Section 2 and Section 3), this confirms the hypothesis of the each column having a constrained end. Some lateral columns, like Column 6, were dramatically dug during the fire and present important cross section reduction on the fire exposed side; instead, the other side presents negligible damage. The central Column 5 and Column 8, exposed on the whole perimeter, present very few centre of gravity positional variations: less than $10 \mathrm{~mm}$ in the latter case and less than 5 in the former.

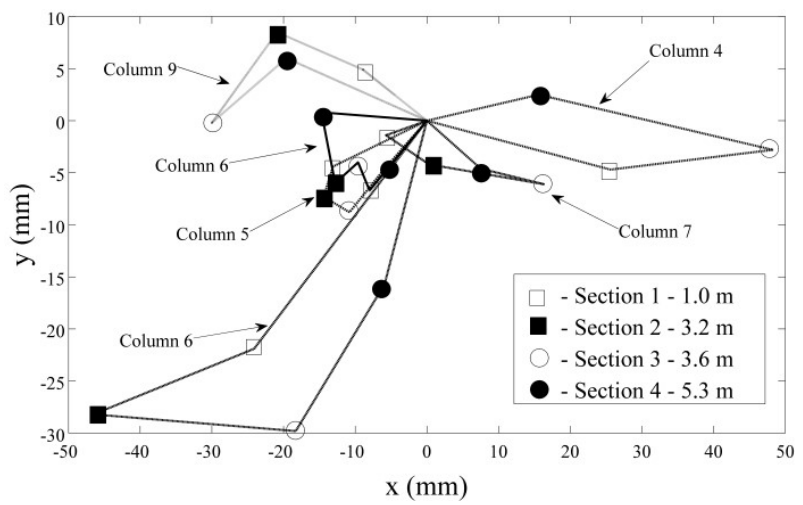

Figure 18: Centre of gravity position of columns cross section at different heights

The fire also damaged the ceiling of the ground floor which consists of transversal $\mathrm{T}$-shaped beams (labeled with $\mathrm{T}$ code in Fig. 16) simply supported directly by the columns and by the longitudinal omega-shaped beams (labeled with L code in Figure 15), supported by the above mentioned T-shaped beams. The maximum deflection recorded (Fig. 18) for each beam is reported in Table 1. The negative sign indicates upward deflection, while the positive one refers to downward deflection. The higher negative deflections for T-shaped beams (corresponding to code T, see Figure 15) are located in the right side of the plant (beams T2-T4-T6) while the other beams (T1T3-T5-T7-T9) present a positive one (see Figure 16). In order to explain these results it must be highlighted that each beam is designed with a negative deflection due to the prestressing tendons. So, in some cases the local damages due to fire were so high to invert the sign of the deflection, in the other cases the negative deflection has survived to fire. The longitudinal omega-shaped beams (corresponding to label L, see Figure 16) present high deflections in the bottom part of the plant (see Figure 16). In particular beam labeled L4 has a maximum deflection of $74.23 \mathrm{~mm}$ corresponding to $6 / 1000$ of the span. This results proves that the fire effect in addition to the dead load of the structure has neglected the initial negative deflection (equal to $25-30 \mathrm{~mm}$, see the undamaged beams labeled La and $\mathrm{Lb}$, present in all L-beams), only in several damaged elements.

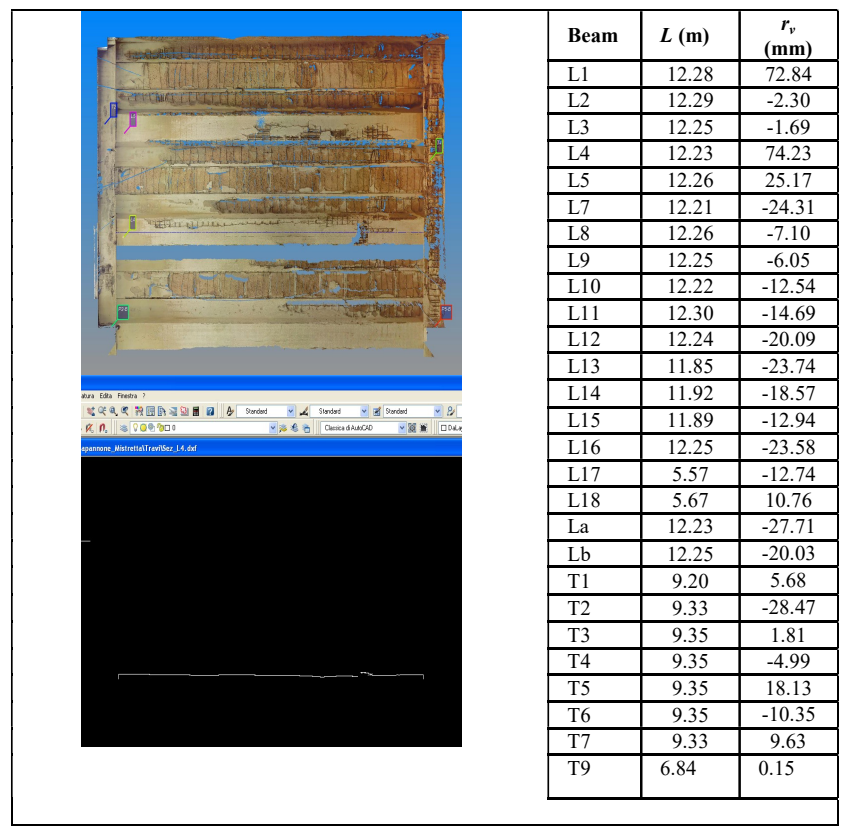

Figure 19: At the right the beam section, at the left the beam span $(L)$ and the deflection $(r v)$ for every beam

The first floor slab deflection was also investigated by means of Laser Scanner and the result is reported in figure 20. In order to assess the deflections caused by the fire, the Reconstructor software was utilized, which generated a horizontal plan passing through an attachment point between the beam and the column not deformed by the fire and, from this, was calculated the deflections with respect to the multitude of points scanned by the laser scanner. Successively, the level curves of the deflections were generated. The maximum recorded deflection was $60 \mathrm{~mm}$. It is located on the left side and in the bottom part of the fired zone. Visible cracks are not present in the extrados, and the unique sign of the fire is the floor discolouration. 


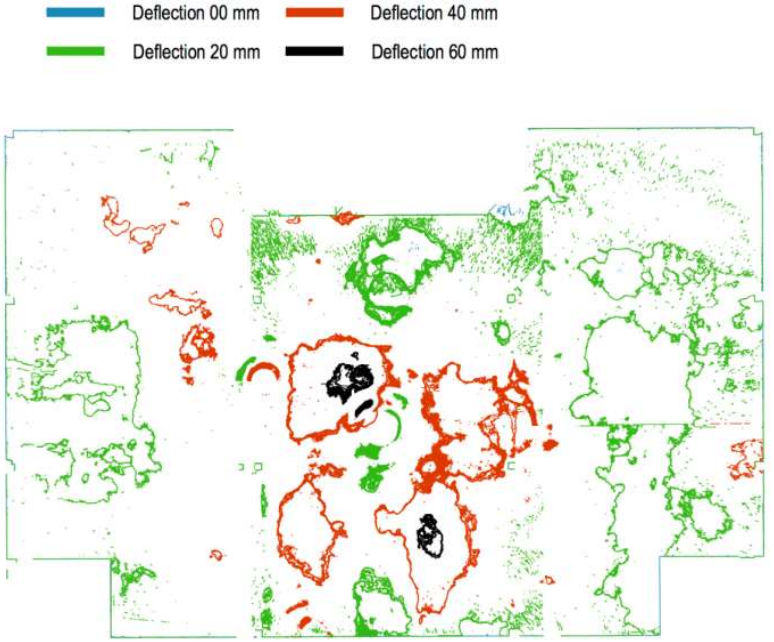

Figure 20: First floor slab deflection

The accurate study of the deformation of the structural elements allowed the assessment of the costs involved in the refurbishment in a much more effective way than the traditional methods used in such cases

\section{CONCLUSION}

The work here presented has looked into and tested the utilization of the terrestrial laser scanner for the monitoring of structures and/or complex buildings. The cases presented have highlighted the advantages and disadvantages of utilizing a scanning technique of this type. In particular, it is important to highlight the following:

- The utilization of the TSL for the monitoring of structures offers a technique which is not destructive, and one which provides the geometry of a building or structure (form and dimensions), even if the latter are inaccessible.

- The possibility of having highly accurate 3D models allows for the study of structural deformations in a very reliable and thorough manner. This gives the opportunity to attentively evaluate the interventions needed in order to restore a structure to its original state, bearing in mind the costs and work times involved.

- One advantage of the TLS is that of having at one's disposition a high number of points that enable more accurate controls and monitoring with respect to the limited number of points that a more traditional type of scanning might have.

Among the disadvantages, we can enumerate: the complex nature of data processing; the difficulty in extracting plans and sections from the 3D model in an automatic manner and without the intervention of an operator. Without doubt the utilization of TLS, for these purposes, offers far more margins for improvement that enable the speeding up of data processing operations and data extraction; as shown in this document.

\section{REFERENCES}

1. F. Segni Pulvirenti, A. Sari, 1994, Architettura tardogotica e d'influsso rinascimentale. Ed. Ilisso, ISBN 88-85098-312
2. F. Stochino, A. Cazzani, S. Poppi, E. Turco, 2015. Sardinia Radio Telescope finite element model updating by means of photogrammetric measurements, Mathematics and mechanics of solids, in press

3. F. Buffa, A. Causin, A. Cazzani, S. Poppi, G. Sanna, M. Solci, F. Stochino, E. Turco, 2015, The Sardinia Radio Telescope: a comparison between close range photogrammetry and fem models, Mathematics and mechanics of solids, in press

4. F. Stochino, G. Carta, 2014. SDOF models for reinforced concrete beams under impulsive loads accounting for strain rate effects, Nuclear Engineering and Design, 276, pp. 7486.

5. S. Tattoni, F. Stochino, F. Caldar, 2013. A simplified approach to serviceability assessment of reinforced concrete structures in fire, Civil-Comp Proceedings, 102.

6. G. Carta , F. Stochino, 2013. Theoretical models to predict the flexural failure of reinforced concrete beams under blast loads, Engineering Structures, 49, pp. 306-315.

7. M. Deidda, A. Dessi, M. Marras, G. Vacca, 2012; Laser scanner survey to cultural heritage conservation and restoration, Int. Arch. Photogramm. Remote Sens. Spatial Inf. Sci., XXXIX-B5, 589-594,

8. M. Acito, F. Stochino, S. Tattoni, 2011. Structural response and reliability analysis of $\mathrm{RC}$ beam subjected to explosive loading, Applied Mechanics and Materials, 82, pp. 434-439.

9. E. Bertacchini, E. Boni, A. Capra, C. Castagnetti, M. Dubbini, 2010. Terrestrial Laser Scanner for Surveying and Monitoring Middle Age Towers. FIG Congress 2010 Facing the Challenges - Building the Capacity Sydney, Australia, 11-16 April 2010

10. González-Aguilera D., Gómez-Lahoz J., Muñoz-Nieto A., Herrero-Pascual J., 2008. Monitoring the health of an emblematic monument from terrestrial laser scanner, Nondestructive Testing and Evaluation, 23:4, 301-315,

11. H.S. Park, H.M. Lee, H. Adeli,I. Lee, 2007 A new approach for health monitoring of structures: terrestrial laser scanning, Comput. Aided Civil Infra.. Eng. 22,19-30.

12. Adami, F. Guerra, P. Vernier, 2007. Laser scanner and architectural accuracy text. XXI International CIPA Symposium, 01-06 October 2007, Athens, Greece.

13. S.J. Gordon, D.D. Lichti, 2007. Modeling terrestrial laser scanner data for precise structural deformation measurement, J. Surv. Eng. 133, pp. 72-80.

14. G. Roberts, C. Brown, X. Meng, 2006 Bridge deflection monitoring: tracking millimeters across the firth of forth, GPS World, pp. 26-31.

15. P. Arias, J. Herraez, H. Lorenzo, and C. Ordon ez,2005. Control of structural problems in cultural heritage monuments using close-range photogrammetry and computer methods, Comput. Struct. J. 83, pp. 1754-1766.

16. Th. Kersten, H. Stenberg, E. Stiemer, 2005. Experiences with terrestrial Laser Scanning for indoor cultural heritage applications using two different scanning system. Proceedings of the ISPRS "Panoramic Photogrammetry Workshop" Berlin, Germany, 24-25/03/2005 IAPRS, Vol. XXVI, Part 5/W8

17. D. Barber, J. Mills, P. Bryan, 2004. Towards a standard specification for terrestrial laser scanning of cultural heritage -one year on. ISPRS, International Archives of Photogrammetry and Remote Sensing, Commission $V$, ISPRS XX Congress, Istanbul, Turchia.

18. L. Bornaz, F. Rinaudo, 2004. Terrestrial laser scanner data Processing. International Archives of Photogrammetry and Remote Sensing, ISPRS. XX Congress, Istanbul, Turchia. 